\title{
Review of the African distribution of the brine shrimp genus Artemia
}

\author{
H Kaiser ${ }^{1 *}$, AK Gordon ${ }^{2}$ and TG Paulet ${ }^{1}$ \\ ${ }^{1}$ Department of Ichthyology and Fisheries Science, Rhodes University, PO Box 94, Grahamstown 6140, South Africa \\ 2 Unilever Centre for Environmental Water Quality, Institute for Water Research, Rhodes University, PO Box 94 ,
}

Grahamstown 6140, South Africa

\begin{abstract}
Brine shrimp (genus Artemia) are small ( 8 to $12 \mathrm{~mm}$ long) cosmopolitan crustaceans (Anostraca) found predominately in hypersaline water bodies such as inland salt lakes and pans, coastal lagoons, and salt works at salinity levels above $40 \mathrm{~g} \cdot \ell^{-1}$. They have been extensively studied due to their high monetary value as food for larval fish in aquaculture and their unique reproductive strategies. Brine shrimp occur as either bisexual species or as parthenogenetic populations. Despite published reviews of their world-wide distribution little is known about their occurrence in Africa. This review adds new information about 70 African Artemia sites and lists 26 potential sites and their coordinates. Sixteen sites in Southern Africa and Namibia were visited during a collecting trip, and new information on the reproductive mode of nine of these sites is given. Several South African populations exhibit bisexual reproduction. In Namibia there are two parthenogenetic populations (Walvis Bay and Swartkops) and an additional bisexual population (Hentie's Bay). A mixed population (bisexual and parthenogenetic reproduction at the same site) was found at Coega, South Africa.
\end{abstract}

Keywords: Biogeography, brine shrimp, site description, hypersaline water bodies

\section{Introduction}

Brine shrimp of the genus Artemia (Crustacea, Anostraca) are of interest to both biologists studying their evolution and developmental biology (Abatzopoulos et al., 2002) and aquaculturists using them as live food in fish and shrimp larviculture (Dhont and Sorgeloos, 2002). The life cycle of Artemia can begin as an embryo within a dormant cyst. Depending on environmental conditions, embryos can enter into diapause and arrested development for many years and are capable of surviving a very wide range of environmental conditions (Clegg and Trotman, 2002). Artemia nauplii can be easily hatched from cysts and have various applications in aquaculture, for example: they can be enriched with nutrients, i.e., essential fatty acids and vitamins to improve their nutritional value to cultured fish larvae or juveniles (Dhont and Sorgeloos, 2002); they have been used as carriers of spawning hormones to treat fish diseases or induce spawning in adult fish (Burton et al., 1998); and they have been tested as a promising vehicle for probiotics in marine fish larviculture (King, 2002).

The genus is cosmopolitan and comprises both sexually reproducing species and parthenogenetic populations. Salinity is the most important environmental factor governing Artemia distribution with populations being found in salt lakes and pans at salinity levels above approximately $40 \mathrm{~g} \cdot \ell^{-1}$ (Vanhaecke et al., 1987) where fish and many predatory invertebrates are absent (Browne and MacDonald, 1982). Information from reviews (Persoone and Sorgeloos, 1980; Browne and MacDonald, 1982; Vanhaecke et al., 1987 and Trianta-

\footnotetext{
* To whom all correspondence should be addressed.

욜 +27466038415 or +27836661439 ; fax: +27466224827 ;

e-mail: h.kaiser@ru.ac.za or hkaiser007@yahoo.com

Received 24 November 2005; accepted in revised form 15 March 2006.
}

phyllidis et al., 1998) suggests that reproduction is sexual in the new world/western hemisphere (Americas), while in the old world (Europe, Asia, and Africa) Artemia populations can reproduce either sexually or parthenogenetically (Browne and MacDonald, 1982).

Artemia cysts can be naturally dispersed over long distances by becoming attached to the feathers of wading birds (Green et al., 2005) or being carried by wind. However, due to their high commercial value, Artemia cysts have also been inoculated into salt pans throughout the world, for example in Kenya (Rasowo and Radull, 1986) and Vietnam (Vu Do Quynh and Nguyen, 1987). Unfortunately, inoculation harbours the danger of introducing invasive species that may establish themselves in the new environment and replace local species. For instance, Van Stappen (2002) suggested that Artemia franciscana may replace other species, such as $A$. salina which is known to occur on the African continent from Tunisia to Southern Africa.

Despite their wide distribution, very little is known about the distribution of Artemia in Southern Africa (Van Stappen, 2002). For example, Persoone and Sorgeloos (1980) listed nine Artemia sites for sub-Saharan Africa, a number that was increased to only 15 sites 18 years later in a review by Triantaphyllidis et al. (1998). In comparison, the number of records for China increased from 2 to 73 sites and in Central America from 18 to 57 (Van Stappen, 2002). Thus, for more than 20 years little progress has been made in our understanding of African Artemia distribution. There is, however, an increasing interest in Artemia research in Africa (Triantaphyllidis et al., 1998). This review contributes to the biogeography of Artemia by presenting information about the presence of Artemia in Africa. We report new sites visited and/or sampled as part of a sampling trip through South Africa and Namibia. Hence, the lists previously provided by Triantaphyllidis et al. (1998) and Van Stappen (2002) were updated. 


\section{Methods}

The sampling trip through parts of South Africa and Namibia was conducted in July/August 2003. Salt pans were visited and GIS coordinates were recorded. Either live Artemia or Artemia cysts were collected. Adult Artemia were preserved in alcohol $\left(>95 \%\right.$ ethanol) and kept frozen at $-20^{\circ} \mathrm{C}$. Cysts were cleaned and dried and kept at $-20^{\circ} \mathrm{C}$ and used for genetic analyses. The approximate size of the pans and the water depth (m) at the time of sampling were estimated, and the salinity $\left(\mathrm{g} \cdot \ell^{-1}\right)$ was recorded using an Atago Hand Refractometer (Model S-10E). Annual average temperature records were taken from information available in GIS databases pertaining to the respective coordinates. Adult Artemia were sexed by using a dissection microscope to discern the egg sacs of females or the claspers of males.

\section{Results and discussion}

\section{Artemia populations in Africa}

Most African Artemia populations have been recorded from countries bordering the Mediterranean (Table 1). Although the

\begin{tabular}{|c|c|c|c|c|c|c|}
\hline \multicolumn{7}{|c|}{ TABLE 1} \\
\hline Country & & Locality & $\begin{array}{l}\text { Geographical } \\
\text { coordinates }\end{array}$ & $\begin{array}{l}\text { Reproductive } \\
\text { mode/spe- } \\
\text { cies }\end{array}$ & $\begin{array}{l}\text { Artemia research } \\
\text { centre number }\end{array}$ & $\begin{array}{c}\text { Reference } \\
\text { number }\end{array}$ \\
\hline \multirow[t]{18}{*}{ Algeria } & \# & $?$ & & & $1285^{*}, 1182^{*}, 1127^{*}, 1098^{*}, 1066^{*}, 1065^{*}$ & 2 \\
\hline & & Chegga Oase & $34^{\circ} 29^{\prime} \mathrm{N}-05^{\circ} 53^{\prime} \mathrm{E}$ & & & 1 \\
\hline & & Chott Djeloud & $34^{\circ} 03^{\prime} \mathrm{N}-06^{\circ} 20^{\prime} \mathrm{E}$ & & & 1 \\
\hline & \# & Chott Merouan & $34^{\circ} 00^{\prime} \mathrm{N}-06^{\circ} 10^{\prime} \mathrm{E}$ & $\mathrm{B}$ & & 3 \\
\hline & & Chott Ouargla & $31^{\circ} 57^{\prime} \mathrm{N}-05^{\circ} 20^{\prime} \mathrm{E}$ & & & 14,15 \\
\hline & & Dayet Morselli & $35^{\circ} 30^{\prime} \mathrm{N}-00^{\circ} 46^{\prime} \mathrm{W}$ & & & 15 \\
\hline & \# & El-Menaceria & & & 1097 and $1091^{*}$ & 2 \\
\hline & & Gharabas Lake & $35^{\circ} 35^{\prime} \mathrm{N}-00^{\circ} 25^{\prime} \mathrm{W}$ & & & 1 \\
\hline & \# & Mellaha Guergour El-Amri & $35^{\circ} 59^{\prime} \mathrm{N}-05^{\circ} 15^{\prime} \mathrm{E}$ & B & & 3 \\
\hline & & Salin de Bethioua & & & $1129 *$ & 2,4 \\
\hline & & Sebket Djendli & $35^{\circ} 43^{\prime} \mathrm{N}-06^{\circ} 32^{\prime} \mathrm{E}$ & & & 1 \\
\hline & & Sebket Ez Zemouk & $35^{\circ} 53^{\prime} \mathrm{N}-06^{\circ} 33^{\prime} \mathrm{E}$ & & & 1 \\
\hline & & Sebket Oran & $35^{\circ} 32^{\prime} \mathrm{N}-00^{\circ} 48^{\prime} \mathrm{W}$ & & & 1 \\
\hline & \# & Sebkha Azrew & $35^{\circ} 43^{\prime} \mathrm{N}-00^{\circ} 08^{\prime} \mathrm{W}$ & $\mathrm{B}$ & & 3 \\
\hline & \# & Sebkha d'Arzeut & & & 1119 & 2 \\
\hline & \# & Sebkha N'zouri & $35^{\circ} 50^{\prime} \mathrm{N}-06^{\circ} 35^{\prime} \mathrm{E}$ & $\mathrm{B}$ & & 3 \\
\hline & $\#$ & Sebkha Sidi Bouzian & $35^{\circ} 52^{\prime} \mathrm{N}-00^{\circ} 35^{\prime} \mathrm{E}$ & $\mathrm{B}$ & & 3 \\
\hline & & Tougourd & $33^{\circ} 06^{\prime} \mathrm{N}-06^{\circ} 07^{\prime} \mathrm{E}$ & & & 16 \\
\hline \multirow{2}{*}{$\begin{array}{l}\text { Cape } \\
\text { Verde }\end{array}$} & \# & Santa Maria & & & & 30 \\
\hline & & Cape Verde Islands & & & & 4 \\
\hline \multirow[t]{10}{*}{ Egypt } & \# & Bourg El-Arab & & $\mathrm{P}$ & $1497^{*}, 1146^{*}, 1136^{*}, 1131^{*}, 1063$ & 2,31 \\
\hline & \# & El Max Saline (Alexandria) & & $\mathrm{P}$ & $1142 *, 1132 *, 1115^{*}, 1064 *$ & 2,31 \\
\hline & & Ismailia & $30^{\circ} 36^{\prime} \mathrm{N}-22^{\circ} 15^{\prime} \mathrm{E}$ & & & 1 \\
\hline & & Port Fouad & & $\mathrm{P}$ & & 4 \\
\hline & \# & Port Said & & $\mathrm{P}$ & $1152,1144 *, 1140 *, 1134 *, 1133^{*}, 1114$ & 2 \\
\hline & & Qarun Lake & & $\mathrm{P}$ & $1139^{*}, 1137,1118^{*}$ & $2,4,31$ \\
\hline & \# & Rassoua & & & $1145^{*}, 1143,1141 *, 1138$ & 2 \\
\hline & & Solar Lake (Sinai) & $29^{\circ} 10^{\prime} \mathrm{N}-34^{\circ} 50^{\prime} \mathrm{E}$ & $\mathrm{P}$ & & $4,15,17,18$ \\
\hline & & Wadi Natron & $30^{\circ} 10^{\prime} \mathrm{N}-30^{\circ} 27^{\prime} \mathrm{E}$ & A. salina & $*$ & 1 \\
\hline & $\#$ & Wadi el Natrun & & A. salina $\left(\mathrm{B}^{31}\right)$ & $1290 *, 1147,1117,1026^{*}, 576,358$ & $2,4,6,31$ \\
\hline \multirow[t]{8}{*}{ Kenya } & & Elmenteita & $00^{\circ} 27^{\prime} \mathrm{S}-36^{\circ} 15^{\prime} \mathrm{E}$ & & & 1 \\
\hline & \# & \begin{tabular}{|l} 
Fundisha \\
\end{tabular} & & & $1035^{*}$ & 2 \\
\hline & \# & Fundisha & & A. salina & & 5 \\
\hline & \# & Kensalt Saltworks & & & $1036^{*}$ & 2 \\
\hline & \# & Kensalt Saltworks & & A. franciscana & $1439 *$ & 2 \\
\hline & \# & Kurawa Saltworks & & A. franciscana & $1441^{*}$ & 2 \\
\hline & \# & Kurawa Saltworks & & A. salina & & 5 \\
\hline & \# & Malindi Saltworks & & A. franciscana & $1440 *$ & 2 \\
\hline \multirow[t]{9}{*}{ Libya } & \# & $?$ & & & $339,1437^{*}$ & 2 \\
\hline & \# & Abu-Khammash & & A. salina & & 6 \\
\hline & & Gabr Acun (Fezzan) & $27^{\circ} \mathrm{N}-13^{\circ} \mathrm{E}$ & & & $16,19,21$ \\
\hline & & Mandara & $26^{\circ} 40^{\prime} \mathrm{N}-13^{\circ} 20^{\prime} \mathrm{E}$ & $\mathrm{B}$ & & 16,19 \\
\hline & & Mandara & & $\mathrm{B}$ & 341,1459 & 2 \\
\hline & & \begin{tabular}{|l} 
Quem el Ma \\
\end{tabular} & $26^{\circ} 41^{\prime} \mathrm{N}-13^{\circ} 22^{\prime} \mathrm{E}$ & & & 16,19 \\
\hline & & Ramba-Az-Zallaf (Fezzan) & $27^{\circ} \mathrm{N}-13^{\circ} \mathrm{E}$ & & & 20 \\
\hline & & Trouna & $26^{\circ} 50^{\prime} \mathrm{N}-13^{\circ} 30^{\prime} \mathrm{E}$ & & & 19 \\
\hline & & \begin{tabular}{|l|} 
Trouna \\
\end{tabular} & & & 340 & 2 \\
\hline \multirow{3}{*}{$\begin{array}{l}\text { Mada- } \\
\text { gascar }\end{array}$} & & Ankiembe Saltworks & & $\mathrm{P}$ & $1314 *$ & $2,4,7$ \\
\hline & & Ifaty Saltworks & & A. franciscana & & 4 \\
\hline & & Salins de Diego Suarez & $12^{\circ} 19^{\prime} \mathrm{S}-49^{\circ} 17^{\prime} \mathrm{E}$ & & & 1 \\
\hline $\begin{array}{l}\text { Mocam- } \\
\text { bique }\end{array}$ & & Lagua Quissico & $24^{\circ} 41^{\prime} \mathrm{S}-34^{\circ} 46^{\prime} \mathrm{E}$ & $\mathrm{P}$ & & 23,26 \\
\hline \multirow[t]{8}{*}{ Morocco } & $\#$ & Chemmaiaa & & A. salina & & 6 \\
\hline & & Larache & $35^{\circ} 12^{\prime} \mathrm{N}-02^{\circ} 20^{\prime} \mathrm{W}$ & $\mathrm{P}$ & & 8,22 \\
\hline & & Moulaya Estuary & $35^{\circ} 07^{\prime} \mathrm{N}-02^{\circ} 20^{\prime} \mathrm{W}$ & & & 1 \\
\hline & & Qued Ammafatma & $28^{\circ} 18^{\prime} \mathrm{N}-12^{\circ} 00^{\prime} \mathrm{W}$ & & & 16 \\
\hline & & Qued Chebeica & $28^{\circ} 25^{\prime} \mathrm{N}-11^{\circ} 50^{\prime} \mathrm{W}$ & & & 16 \\
\hline & & Sebket Bon Areg & $35^{\circ} 10^{\prime} \mathrm{N}-02^{\circ} 50^{\prime} \mathrm{W}$ & & & 1 \\
\hline & & Sebket Zima & $32^{\circ} 05^{\prime} \mathrm{N}-08^{\circ} 40^{\prime} \mathrm{W}$ & & & 1 \\
\hline & \# & Souzamma & & & $1427 *$ & 2 \\
\hline
\end{tabular}




\begin{tabular}{|c|c|c|c|c|c|c|}
\hline \multirow[t]{6}{*}{ Namibia } & \# & $?$ & & & $1418^{*}$ & 2 \\
\hline & \# & Henties Bay Salt Refineries & $22^{\circ} 00^{\prime} \mathrm{S}-00^{\circ} 14^{\prime} \mathrm{E}$ & & & 28 \\
\hline & $\#$ & Swakopmund Saltworks & $22^{\circ} 40^{\prime} \mathrm{S}-14^{\circ} 34^{\prime} \mathrm{E}$ & & & 28 \\
\hline & & Vineta Swakopmund & $22^{\circ} 40^{\prime} \mathrm{S}-14^{\circ} 34^{\prime} \mathrm{E}$ & $\mathrm{P}$ & & 1,4 \\
\hline & & Vineta Swakopmund & & $\mathrm{P}$ & $1186^{*}, 480^{*}$, & $2,6,7,9$ \\
\hline & \# & Walvis Bay & $22^{\circ} 56^{\prime} \mathrm{S}-14^{\circ} 30^{\prime} \mathrm{E}$ & & & 28 \\
\hline \multirow{4}{*}{$\begin{array}{l}\text { Niger } \\
\text { Senegal }\end{array}$} & & Teguidda In Tessoun & $17^{\circ} 26^{\prime} \mathrm{N}-06^{\circ} 39^{\prime} \mathrm{E}$ & & & 1 \\
\hline & & Dakar & $14^{\circ} 34^{\prime} \mathrm{N}-17^{\circ} 29^{\prime} \mathrm{W}$ & & & 1 \\
\hline & & Lake Kayar & $14^{\circ} 55^{\prime} \mathrm{N}-17^{\circ} 11^{\prime} \mathrm{W}$ & & & 1 \\
\hline & & Lake Retba & $14^{\circ} 50^{\prime} \mathrm{N}-17^{\circ} 20^{\prime} \mathrm{W}$ & & & 16 \\
\hline \multirow{43}{*}{$\begin{array}{l}\text { South } \\
\text { Africa }\end{array}$} & $\#$ & $?$ & & & $1267,1170,1159$ & 2 \\
\hline & \# & Bloemfontein Saltworks & & $\mathrm{P}$ & & 10 \\
\hline & \# & Brandtvlei Saltworks & $30^{\circ} 22^{\prime} \mathrm{S}-20^{\circ} 13^{\prime} \mathrm{E}$ & & & 29 \\
\hline & \# & Brandtvlei Saltworks & $30^{\circ} 26^{\prime} \mathrm{S}-20^{\circ} 44^{\prime} \mathrm{E}$ & & & 28 \\
\hline & & Coega Salt Flats & $33^{\circ} 46^{\prime} \mathrm{S}-25^{\circ} 40^{\prime} \mathrm{E}$ & $\mathrm{P}$ & & 1 \\
\hline & \# & Driehoekspan & $29^{\circ} 45^{\prime} \mathrm{S}-23^{\circ} 14^{\prime} \mathrm{E}$ & & & 29 \\
\hline & $\#$ & Haaegestad Pan & & & & 30 \\
\hline & $\#$ & Hayfield Saltpan & $29^{\circ} 15^{\prime} \mathrm{S}-24^{\circ} 13^{\prime} \mathrm{E}$ & & & 29 \\
\hline & \# & Holpan Saltworks & $30^{\circ} 22^{\prime} \mathrm{S}-20^{\circ} 30^{\prime} \mathrm{E}$ & & & 29 \\
\hline & \# & Holpan Saltworks & $30^{\circ} 12^{\prime} \mathrm{S}-20^{\circ} 40^{\prime} \mathrm{E}$ & & & 29 \\
\hline & \# & Jonkerwater Saltworks & $30^{\circ} 05^{\prime} \mathrm{S}-22^{\circ} 36^{\prime} \mathrm{E}$ & & & 29 \\
\hline & \# & Kaalpan Saltworks & $30^{\circ} 00^{\prime} \mathrm{S}-20^{\circ} 03^{\prime} \mathrm{E}$ & & & 29 \\
\hline & $\#$ & Klein Soutpan & $30^{\circ} 27^{\prime} \mathrm{S}-22^{\circ} 24^{\prime} \mathrm{E}$ & & & 29 \\
\hline & $\#$ & Klipfontein Saltworks & $32^{\circ} 57^{\prime} \mathrm{S}-18^{\circ} 13^{\prime} \mathrm{E}$ & & & 28 \\
\hline & $\#$ & Kliphoek Salina & & & & 27 \\
\hline & \# & Missionvale Salina & $33^{\circ} 52^{\prime} \mathrm{S}-25^{\circ} 32^{\prime} \mathrm{E}$ & & & 27 \\
\hline & \# & Paternoster Salt Pan & $33^{\circ} 47^{\prime} \mathrm{S}-17^{\circ} 55^{\prime} \mathrm{E}$ & & & 29 \\
\hline & $\#$ & Playas & & & 1169 & 2 \\
\hline & \# & Rietfontein se Pan & $30^{\circ} 16^{\prime} \mathrm{S}-20^{\circ} 07^{\prime} \mathrm{E}$ & & & 29 \\
\hline & $\#$ & Reynekespan Saltworks & $33^{\circ} 41^{\prime} \mathrm{S}-25^{\circ} 47^{\prime} \mathrm{E}$ & & & 28 \\
\hline & $\#$ & Reynekespan ( 2 populations) & $29^{\circ} 43^{\prime} \mathrm{S}-24^{\circ} 15^{\prime} \mathrm{E}$ & & & 29 \\
\hline & \# & Saldanha Steel & $33^{\circ} 00^{\prime} \mathrm{S}-18^{\circ} 02^{\prime} \mathrm{E}$ & & & 28 \\
\hline & $\#$ & Salt Lake & $29^{\circ} 17^{\prime} \mathrm{S}-24^{\circ} 00^{\prime} \mathrm{E}$ & & & 29 \\
\hline & \# & Hopetown Salt Pan & & & $1121^{*}$ & 2 \\
\hline & \# & Hopetown & & & & 30 \\
\hline & \# & Sodium Saltworks & $30^{\circ} 10^{\prime} \mathrm{S}-23^{\circ} 08^{\prime} \mathrm{E}$ & & & 29 \\
\hline & $\#$ & Soutpan area & $28^{\circ} 42^{\prime} \mathrm{S}-26^{\circ} 03^{\prime} \mathrm{E}$ & & & 29 \\
\hline & \# & Soutpan Saltworks & $28^{\circ} 44^{\prime} \mathrm{S}-26^{\circ} 04^{\prime} \mathrm{E}$ & & & 29 \\
\hline & $\#$ & Sundays River Saltworks & $33^{\circ} 38^{\prime} \mathrm{S}-25^{\circ} 43^{\prime} \mathrm{E}$ & & & 28 \\
\hline & & Swartkops & $33^{\circ} 52^{\prime} \mathrm{S}-25^{\circ} 36^{\prime} \mathrm{E}$ & & & 1,2 \\
\hline & & Swartkops & & A. salina. & $1179^{*}$ & 30 \\
\hline & \# & Swartkops Marina & $33^{\circ} 51^{\prime} \mathrm{S}-25^{\circ} 34^{\prime} \mathrm{E}$ & & & 28 \\
\hline & \# & Swartkops Cerebos & $33^{\circ} 47^{\prime} \mathrm{S}-25^{\circ} 32^{\prime} \mathrm{E}$ & & & 28 \\
\hline & \# & Uniesoutpan & $29^{\circ} 36^{\prime} \mathrm{S}-24^{\circ} 26^{\prime} \mathrm{E}$ & & & 29 \\
\hline & & Velddrif & & A. salina & 1289 & 2,13 \\
\hline & $\#$ & Velddrif Saltworks & $32^{\circ} 43^{\prime} \mathrm{S}-18^{\circ} 12^{\prime} \mathrm{E}$ & & & 28 \\
\hline & \# & Velddrif Saltworks & $32^{\circ} 47^{\prime} \mathrm{S}-18^{\circ} 10^{\prime} \mathrm{E}$ & & & 28 \\
\hline & \# & Vermeulenspan & $29^{\circ} 45^{\prime} \mathrm{S}-24^{\circ} 20^{\prime} \mathrm{E}$ & & & 29 \\
\hline & \# & Wintersdam Farm & $28^{\circ} 45^{\prime} \mathrm{S}-26^{\circ} 08^{\prime} \mathrm{E}$ & & & 12 \\
\hline & \# & Witkraal Saltpan & $28^{\circ} 58^{\prime} \mathrm{S}-25^{\circ} 31^{\prime} \mathrm{E}$ & & & 29 \\
\hline & \# & Witpan Saltworks & $29^{\circ} 53^{\prime} \mathrm{S}-24^{\circ} 03^{\prime} \mathrm{E}$ & & & 29 \\
\hline & \# & Yzerfontein Saltworks & $33^{\circ} 19^{\prime} \mathrm{S}-18^{\circ} 10^{\prime} \mathrm{E}^{\prime}$ & & & 28 \\
\hline & \# & Zoutaar Saltworks & $30^{\circ} 19^{\prime} \mathrm{S}-23^{\circ} 05^{\prime} \mathrm{E}$ & & & 29 \\
\hline \multirow[t]{13}{*}{ Tunisia } & \# & ? & & & $465,2,3$ & 2 \\
\hline & & Bekalta & $36^{\circ} 48^{\prime} \mathrm{N}-10^{\circ} 20^{\prime} \mathrm{E}$ & A. salina & * & 24 \\
\hline & & Bekalta & & A. salina & $363^{*}$ & 2 \\
\hline & & Chott Ariana & $36^{\circ} 54^{\prime} \mathrm{N}-10^{\circ} 18^{\prime} \mathrm{E}$ & A. salina & $*$ & 24,25 \\
\hline & & Chott Ariana & & A. salina & $360^{*}$ & 2 \\
\hline & & Chott El Dierid & $33^{\circ} 42^{\prime} \mathrm{N}-08^{\circ} 26^{\prime} \mathrm{E}$ & & & 1 \\
\hline & & Megrine & $36^{\circ} 47^{\prime} \mathrm{N}-10^{\circ} 14^{\prime} \mathrm{E}$ & A. salina & * & 24 \\
\hline & & Megrine & & A. salina & $1268^{*}, 361,466^{*}$ & 2 \\
\hline & & Sebket Kowezia & $36^{\circ} 26^{\prime} \mathrm{N}-09^{\circ} 46^{\prime} \mathrm{E}$ & & $*$ & 1 \\
\hline & & Sebket Mta Moknine & $35^{\circ} 39^{\prime} \mathrm{N}-10^{\circ} 53^{\prime} \mathrm{E}$ & A. salina & * & 24 \\
\hline & & Sebket Sidi El Hani & $35^{\circ} 31^{\prime} \mathrm{N}-10^{\circ} 27^{\prime} \mathrm{E}$ & & * & 1 \\
\hline & \# & Sfax & $35^{\circ} 45^{\prime} \mathrm{N}-10^{\circ} 43^{\prime} \mathrm{E}$ & A. salina & * & 24,32 \\
\hline & & Sfax & & A. salina & $1493 *, 1492 *, 1269 *, 506,362$ & 2,9 \\
\hline
\end{tabular}

Legend: $(B)=$ bisexual but species not known; $(P)=$ parthenogenetically reproducing population; ${ }^{*}=$ cysts are available at the Artemia Reference Center (ARC), Ghent, Belgium; \# = a new Artemia population added to the list since the last general review by Triantaphyllidis et al. (1998).

The number in the last column indicates the reference in which the Artemia population was first reported; ? Site name not known or not given.

\section{References for Table 1}

1. Vanhaecke et al. (1987); 2. ARC table; 3. Zemmouri (1991); 4. Triantaphyllidis et al. (1998); 5. Rasowo and Radull (1986); 6. Triantaphyllidis et al. (1997b); 7. Triantaphyllidis et al. (1996); 8. Hontoria and Amat (1992); 9. Triantaphyllidis et al. (1997a); 10. Williams and Mitchell (1992); 11. Seaman et al. (1991); 12. Mitchell and Seaman (1988); 13. Amat et al. (1995); 14. Cole and Brown (1967) cited in Vanhaecke et al. (1987); 15. McCarraher (1972) cited in Vanhaecke et al. (1987); 16. Dumont (1979) cited in Vanhaecke et al. (1987); 17. Por (1968) cited in Vanhaecke et al. (1987); 18. Por (1969) cited in Vanhaecke et al. (1987); 19. Monod (1969) cited in Vanhaecke et al. (1987); 20. Ghannudi and Tufail (1978) cited in Vanhaecke et al. (1987); 21. Beadle (1974) cited in Vanhaecke et al. (1987); 22. Amat Domenech (1980) cited in Vanhaecke et al. (1987); 23. De Pinho Canelhas (1971) cited in Vanhaecke et al. (1987); 24. Van Ballaer et al. (1987) cited in Vanhaecke et al. (1987); 25. Clark and Bowen (1976) cited in Vanhaecke et al. (1987); 26. Sousa (1994) cited in Triantaphyllidis et al. (1998); 27. Du Toit (2001); 28. Present study - cyst/nauplii samples collected.; 29. Present study - personal communication; 30. Van Stappen (2002); 31. Baxevanis et al. 2004. 32. Toumi et al. (2005). 


\begin{tabular}{|c|c|c|c|c|}
\hline \multicolumn{5}{|c|}{$\begin{array}{c}\text { TABLE } 2 \\
\text { Abiotic characteristics of } 16 \text { sites from South Africa }{ }^{\mathrm{a}} \text { and } \\
\text { Namibia }^{\mathrm{b}} \text { from which adult Artemia and/or Artemia cysts } \\
\text { were collected during July/August } 2003\end{array}$} \\
\hline Site name & Type & $\begin{array}{l}\text { Elevation } \\
(\mathrm{m})\end{array}$ & \begin{tabular}{|c|} 
Approximate \\
size $\left(\mathbf{k m}^{2}\right)$
\end{tabular} & $\begin{array}{l}\text { Temperature in }{ }^{\circ} \mathrm{C} \\
\text { (average; } \mathrm{min} / \mathrm{max} \text { ) }\end{array}$ \\
\hline Brandtvlei $^{\mathrm{a}}$ & $\mathrm{I}$ & 940 & 4 & $20.2 ;-8 /+42$ \\
\hline Coega Cerebos $^{\mathrm{a}}$ & $\mathrm{C}$ & 1 & 10 & $19.1 ; 0 /+32$ \\
\hline Henties Bay ${ }^{\mathrm{b}}$ & $\mathrm{C}$ & 0 & 5 & $20 ; 0 /+34$ \\
\hline Kleinzee Yacht Club ${ }^{\mathrm{a}}$ & $\mathrm{N}$ & 0 & 3 & $? ; 0 /+40$ \\
\hline Klipfontein Salt ${ }^{\mathrm{a}}$ & $\mathrm{C}$ & 15 & 5 & $23.6 ; 0 /+40$ \\
\hline Missionvale Salt ${ }^{\mathrm{a}}$ & $\mathrm{C}$ & 4 & 5 & $18.3 ; 0 /+35$ \\
\hline Reynekespan $^{\mathrm{a}}$ & $\mathrm{I}$ & 1149 & 0.5 & $22.2 ;-6 /+40$ \\
\hline Saldanha Steel $^{\mathrm{a}}$ & $\mathrm{S}$ & 40 & 0.5 & $23.6 ; 0 /+40$ \\
\hline Sundays River $^{\mathrm{a}}$ & $\mathrm{I}$ & 30 & 5 & $20.2 ;-1 /+36$ \\
\hline Swakopmund ${ }^{\mathrm{b}}$ & $\mathrm{C}$ & 0 & 4 & $20 ; 0 /+34$ \\
\hline Swartkops Cerebos $^{\mathrm{a}}$ & $\mathrm{I}$ & 10 & 4 & $18.8 ; 0 /+35$ \\
\hline Swartkops Salt ${ }^{\mathrm{a}}$ & I & 3 & 4 & $18.2 ; 0 /+35$ \\
\hline Velddrif Cerebos $^{\mathrm{a}}$ & $\mathrm{C}$ & 12 & 5 & $24.6 ; 0 /+40$ \\
\hline Velddrif Salt $^{\mathrm{a}}$ & $\mathrm{C}$ & 12 & 5 & $24.6 ; 0 /+40$ \\
\hline Walvis Bay ${ }^{\mathrm{b}}$ & $\mathrm{C}$ & 0 & 10 & $20 ; 0 /+34$ \\
\hline Yzerfontein $^{\mathrm{a}}$ & $\mathrm{S}$ & 80 & 0.5 & $20 ;-2 /+38$ \\
\hline
\end{tabular}

Legend:

$I=$ Inland salt works; $C=$ Coastal salt works; $N=$ Natural coastal lagoon; $S=$ Salt pan.

climate of this region is well suited to the development of an Artemia biotope (Vanhaecke et al., 1987), the high number of records for this region is probably a result of a relatively higher collection effort.

The reproductive mode for most Artemia populations in Algeria has not been established. Populations with a confirmed reproductive mode are bisexual, but their species status is either not known or has not been published. One bisexual population was recorded at a salt works and others were found in natural salt lakes (Zemmouri, 1991). In Libya one population was confirmed as bisexual (Triantaphyllidis et al., 1997b), but the reproductive mode of the other populations was not given. Records from Morocco show one population of A. salina (Triantaphyllidis et al., 1997b), one occurrence of a parthenogenetic population, and populations of unknown reproductive mode. Most Egyptian Artemia populations are parthenogenetic. A. salina was found at the Wadi Natron site and a permanent population of A. franciscana was discovered at a salt works (Triantaphyllidis et al., 1998). All Tunisian populations have been reported as A. salina. Dumont (1979) reported an Artemia population from Lake Retba, Senegal. Only personal communications account for the records of all other Artemia populations in Niger and Senegal (Table 1), and their reproductive mode is not known.

Records available prior to our sampling trip show the existence of both bisexual species and parthenogenetic populations in sub-Saharan Africa (Table 1). These populations were located at salt works, some of which were operating at the time of sampling, others had been abandoned. In addition, a number of personal communications report the existence of Artemia populations which required further investigation. For example, for three of six South African populations listed by Van Stappen (2002) the information is based on personal communications, thus their species status was undetermined. Artemia salina is reported to have been inoculated into Kenyan salt works (Rasowo and Radull, 1986; see Table 1), but the Artemia Reference Centre in Ghent, Belgium, identified Artemia from those salt works as A. franciscana. Both Mozambique and Namibia have partheno- genetic Artemia populations occurring at salt works. A parthenogenetic population as well as A. franciscana have been found in Madagascar, with an unidentified population occurring in the northern part of the island. The A. franciscana population was not permanent, and had to be inoculated annually (Triantaphyllidis et al., 1998).

The low number of recorded Artemia populations in Africa does not reflect Artemia distribution on the continent. Large areas of Africa, (i.e., Mauritania, Somalia, Ethiopia, Sudan, and southern Africa) are considered potential Artemia habitat (Vanhaecke et al., 1987). However, very few collections have been reported for these areas. Vanhaecke et al. (1987) suggested that 97\% of the known Artemia populations in Africa are in areas where yearly evaporation exceeds yearly precipitation. Williams (1996) estimated that $37 \%\left(11.3 \times 10^{6} \mathrm{~km}^{2}\right)$ of the African continent can be categorised as arid and semi-arid where salt lakes are likely to occur.

Salt lakes of northern Africa were among the first on the continent to be investigated, followed by locations in East Africa (Hammer, 1986; Seaman et al., 1991). More recently, the salt pans of Southern Africa have come under investigation (Seaman et al., 1991). Studies of these saltwater bodies have mainly focused on geological and chemical characteristics, with few biological surveys. Thus, the presence or absence of Artemia in most of these water bodies remains unclear. Saline water bodies in East and Southern Africa with salinities greater than $40 \mathrm{~g} \cdot \ell^{-1}$ (Table 3) may contain populations of Artemia and should be the focus of further investigations. Lists of salt pans or lakes with salinities below $40 \mathrm{~g} \cdot \ell^{-1}$ can be found in Seaman et al. (1991), Hammer (1986), Shumway (1999) and Williams (1996).

\section{Addition of new Artemia sites in sub-Saharan Africa}

During July and August 2003, adult Artemia and cysts were collected from nine and five sites, respectively. For most adult specimens gender could be determined, but as the sample sizes 


\begin{tabular}{|c|c|c|c|c|}
\hline \multicolumn{5}{|c|}{ 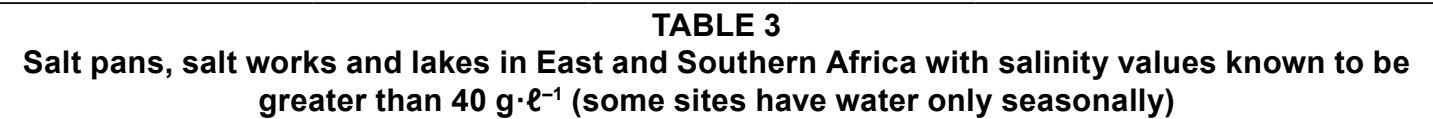 } \\
\hline Name & Position & Country & Salinity & Reference \\
\hline Annaspan $^{\mathrm{b}}$ & $28^{\circ} 31^{\prime} \mathrm{S}-25^{\circ} 48^{\prime} \mathrm{E}$ & South Africa & & Present study \\
\hline Banksdrifpan $^{\mathrm{b}}$ & $28^{\circ} 56^{\prime} \mathrm{S}-25^{\circ} 14^{\prime} \mathrm{E}$ & South Africa & & Present study \\
\hline Bogoria & - & Kenya & 50 & Williams (1996) \\
\hline Britten Pan & $27^{\circ} 45^{\prime} \mathrm{S}-25^{\circ} 21^{\prime} \mathrm{E}$ & South Africa & 181.6 & Seaman et al. (1991) \\
\hline Dealsville $^{\mathrm{b}}$ & $28^{\circ} 40^{\prime} \mathrm{S}-25^{\circ} 41^{\prime} \mathrm{E}$ & South Africa & & Present study \\
\hline Delareyville area $^{\mathrm{a}}$ & $26^{\circ} 41^{\prime} \mathrm{S}-25^{\circ} 27^{\prime} \mathrm{E}$ & South Africa & & Present study \\
\hline Etosha Pan & $18^{\circ} 40^{\prime} \mathrm{S}-16^{\circ} 40^{\prime} \mathrm{E}$ & Namibia & 62.0 & Seaman et al. (1991) \\
\hline Florisbad Pan $^{\mathrm{a}}$ & $28^{\circ} 45^{\prime} \mathrm{S}-26^{\circ} 05^{\prime} \mathrm{E}$ & South Africa & 197.3 & Seaman et al. (1991); Present study \\
\hline Gannaleegte $(3 \text { sites })^{\mathrm{a}}$ & $28^{\circ} 42^{\prime} \mathrm{S}-25^{\circ} 52^{\prime} \mathrm{E}$ & South Africa & & Present study \\
\hline Holfontein $^{\mathrm{a}}$ & $28^{\circ} 42^{\prime} \mathrm{S}-25^{\circ} 52^{\prime} \mathrm{E}$ & South Africa & & Present study \\
\hline Hosabes Pool & $23^{\circ} 30^{\prime} \mathrm{S}-15^{\circ} 05^{\prime} \mathrm{E}$ & Namibia & $115.9-161.7$ & Seaman et al. (1991) \\
\hline Karee Pan $^{\mathrm{b}}$ & $27^{\circ} 30^{\prime} \mathrm{S}-25^{\circ} 35^{\prime} \mathrm{E}$ & South Africa & 41.8 & Seaman et al. (1991); Present study \\
\hline 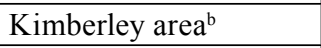 & $28^{\circ} 53^{\prime} \mathrm{S}-24^{\circ} 15^{\prime} \mathrm{E}$ & South Africa & & Present study \\
\hline Koppieskraal Pan & $26^{\circ} 55^{\prime} \mathrm{S}-20^{\circ} 18^{\prime} \mathrm{E}$ & South Africa & 48.6 & Seaman et al. (1991) \\
\hline Magadi & - & Kenya & 114 & Williams (1996) \\
\hline Mollerspan $^{\mathrm{b}}$ & $28^{\circ} 53^{\prime} \mathrm{S}-24^{\circ} 14^{\prime} \mathrm{E}$ & South Africa & & Present study \\
\hline Natron & - & Tanzania & 340 & Williams (1996) \\
\hline Oranjemund Pan & $28^{\circ} 35^{\prime} \mathrm{S}-16^{\circ} 35^{\prime} \mathrm{E}$ & Namibia & 302.4 & Seaman et al. (1991) \\
\hline Rensburg Salt Pan & $28^{\circ} 55^{\prime} \mathrm{S}-26^{\circ} 05^{\prime} \mathrm{E}$ & South Africa & 102.4 & Seaman et al. (1991) \\
\hline Skietbaana $^{\mathrm{a}}$ & $26^{\circ} 43^{\prime} \mathrm{S}-25^{\circ} 28^{\prime} \mathrm{E}$ & South Africa & & Present study \\
\hline Skoppan $^{\mathrm{b}}$ & $28^{\circ} 40^{\prime} \mathrm{S}-26^{\circ} 05^{\prime} \mathrm{E}$ & South Africa & 160.1 & Seaman et al. (1991); Present study \\
\hline Soutbron $^{\mathrm{a}}$ & $28^{\circ} 41^{\prime} \mathrm{S}-25^{\circ} 50^{\prime} \mathrm{E}$ & South Africa & & Present study \\
\hline Soutpan area $(15 \text { sites })^{\mathrm{a}}$ & $28^{\circ} 42^{\prime} \mathrm{S}-26^{\circ} 03^{\prime} \mathrm{E}$ & South Africa & & Present study \\
\hline Stink Pan & $27^{\circ} 46^{\prime} \mathrm{S}-26^{\circ} 40^{\prime} \mathrm{E}$ & South Africa & 51.1 & Seaman et al. (1991) \\
\hline Tara $^{\mathrm{a}}$ & $28^{\circ} 42^{\prime} \mathrm{S}-25^{\circ} 49^{\prime} \mathrm{E}$ & South Africa & & Present study \\
\hline Wadrif Soutpan $^{\mathrm{a}}$ & $32^{\circ} 12^{\prime} \mathrm{S}-18^{\circ} 21^{\prime} \mathrm{E}$ & South Africa & & Present study \\
\hline
\end{tabular}

were low, only numbers of males and females in parentheses, rather than percentage values, will be reported. These results can be used as an indication of the most likely reproductive status of the population since parthenogenetic populations do not have males, except for the rare occurrence of non-functional males (MacDonald and Brown, 1990). Therefore, these figures do not allow an estimation of the gender ratio in the population as this would require a larger sample size and repeated sampling at different times. They provide, however, a basis for hypotheses regarding a population's reproductive mode.

In South Africa the sites Brandtvlei (10:47), Yzerfontein (13:33), Reynekespan (30:17), Swartkops Marina Salt (9:5), Swartkops Cerebos (7:7) and Missionvale (3:10) were inhabited either by sexually reproducing Artemia only, or they had at least one bisexual species. The presence of males in a population suggests the existence of a bisexual species, but the same site may also contain a parthenogenetic population. For example, Van Stappen (2002) listed the population from Coega saltworks as parthenogenetic. Unpublished work using genetic markers confirmed that this site had both a sexually reproducing species and parthenogenetic component. Genetic analyses are required to distinguish populations at one site from each other and to identify species. We suggest that the six South African populations exhibited sexual reproduction and, except for the population at Velddrif described by Amat et al.(1995), this is the first survey to include these South African populations.
At two sites in Namibia no males were recorded. These sites were Swakopmund (0:30) and Walvis Bay Salt Pan (0:42). Previously recorded Namibian populations (see Table 1) were parthenogenetic, and our recent records from the population at Swakopmund suggested the existence of a parthenogenetic population, thus confirming this reproductive mode for both sites using samples taken in 2003. Results from collections at Henties Bay (14:5) provide the first record of bisexual reproduction for Namibian Artemia although the species status of this population has not yet been determined.

Additional information (Table 2) was collected for some new southern African Artemia sites investigated as part of this study. This information included site management, GIS data relating average temperatures and their ranges, as well as elevation and the approximate area of the sites at the time of sampling. The latter value fluctuates strongly depending on season and rainfall and records should be taken repeatedly during the year.

Several other sites were visited but no Artemia or cysts were found (see Table 3). Of the 47 sites in South Africa and Namibia, 24 were at an elevation of above $1000 \mathrm{~m}$, with the highest elevation being $1556 \mathrm{~m}$ a.m.s.l. Four sites were at sea level. The average elevation of the other sites was $793 \mathrm{~m}$ a.m.s.l. The average annual ambient temperature was $18.2^{\circ} \mathrm{C}$ $\left(-8\right.$ to $\left.+42^{\circ} \mathrm{C}\right)$. The average minimum and maximum ambient temperatures of these 47 Southern African sites were $-0.4^{\circ} \mathrm{C}$ and $36^{\circ} \mathrm{C}$, respectively. 


\section{Site names}

There are two South African populations at Swartkops; these are at Swartkops Marina and Swartkops Cerebos. These sites belong to different salt production companies and, although geographically close to each other, were not listed as being the same due to different management. Similarly, sites listed under the name Velddrif do not all belong to the same salt company. In addition, very similar names have been given to different sites; for example, the name Soutpan (Afrikaans for salt pan) appears as part of several composite names. Spelling may differ between Afrikaans and English (i.e., Coega or Koega)

\section{Conclusion}

There are 127 records of either sexually reproducing or parthenogenetic populations of Artemia on the continent, but reproductive mode has only been given for 41 populations. An estimated $32 \%$ of the populations appear to be parthenogenetic and $68 \%$ are bi-sexual species. However, only a tentative species list can be presented due to the uncertainty and paucity of published and anecdotal information. Most work on African Artemia populations needs verification using molecular techniques. Thus, applying caution with regard to the precision of the data, and considering that relatively few records are available, it is estimated that about $50 \%$ of the identified populations are $A$. salina, $12 \%$ can be hypothetically assigned to A. franciscana, and $38 \%$ of the populations so far described appear to be parthenogenetically reproducing Artemia.

By combining the information obtained during the sampling trips and climatic and GIS data available for South Africa with information provided in the literature the number of potentially available Artemia biotopes was updated (Table 3). These sites deserve further investigation. In order to obtain a representative picture of South African populations, sampling should be done repeatedly over several years and throughout the year since distinct dry seasons occur.

\section{Acknowledgements}

This study was supported by the INCO project on Artemia Biodiversity (Project Number ICA4-CT-2001-10020), an International Scientific Cooperation Programme with Developing Countries of the European Commission. We also acknowledge funding from the Rhodes University Joint Research Council Fund.

\section{References}

ABATZOPOULOS ThJ, BEARDMORE JA, CLEGG JS and SORGELOOS P (2002) Artemia Basic and Applied Biology. Kluwer Acadamic Publishers, Dordrecht / London / Boston. 286 pp.

AMAT DOMENECH F (1980) Differentiation in Artemia strains from Spain. In: Sorgeloos P, Roels $\mathrm{O}$ and Jaspers E (eds.) The Brine Shrimp Artemia. Vol. 1. Morphology, Genetics, Radiobiology, Toxicology. Universal Press, Wetteren Belgium. 19-41.

AMAT F, BARATA C and HONTORIA F (1995) A Mediterranean origin for the Velddrif (South Africa) Artemia salina population. J. Biogeogr. 22 49-59.

BAXEVANIS AD, EL-BERMAWI $\mathrm{N}$ and ABATZOPOULOS, ThJ (2004) Salinity effects on maturation, reproductive and life span characteristics of four Egyptian Artemia populations (International Study on Artemia. LXVIII). Hydrobiol. 513 87-100.

BEADLE LC (1974) Temporary saline and thermal waters: Lake Chilwa. In: Urban EK, Fry CH and Keith S (eds.) The Inland Waters of Tropical Africa: An Introduction to Tropical Limnology. Longman, London and New York. 259-282.

BROWNE RA and MACDONALD GH (1982) Biogeography of the brine shrimp Artemia: Distribution of parthenogenetic and sexual populations. J. Biogeogr. $9331-338$.

BURTON S, KAISER H and HECHT T (1998) The potential of Artemiamediated delivery of a gonadotropin hormone analogue to induce ovulation in the cardinal tetra (Paracheirodon axelrodi). Aquarium Sci. Cons. 2 89-92.

CLARK LS and BOWEN ST (1976) The genetics of Artemia salina VII. Reproductive isolation. J. Hered. 67 385-388.

CLEGG JS and TROTMAN CNA (2002) Physiological and biochemical aspects of Artemia ecology. In: Abatzopolous Th J, Beardmore JA, Clegg JS and Sorgeloos (eds.) Artemia Basic and Applied Biology. Kluwer Acadamic Publishers, Dordrecht/London/Boston. 129-170.

COLE GA and BROWN RJ (1967) The chemistry of Artemia habitats. Ecol. 48 858-861.

DE PINHO CANELHAS MH (1971) Breve notica sobre a Artemia na lagoa Nhamaiane IX Jornadas Silvo Agronomicas Sept 27-Oct 2. Laurnco Marques 267.

DHONT J and SORGELOOS P (2002) Applications of Artemia. In: Abatzopolous ThJ, Beardmore JA, Clegg JS and Sorgeloos (eds.) Artemia Basic and Applied Biology. Kluwer Acadamic Publishers, Dordrecht/London/Boston. 251-257.

DU TOIT SR (2001) Biological Management of South African Solar Saltworks. Ph.D. Thesis University of Port Elizabeth, South Africa.

DUMONT HJ (1979) Limnologie van Sahara en Sahel: Naar een Beter Begrip van be Klimaatsveranderingen van het laat Pleistoceen en Holoceen. Thesis, State University, Ghent. Belgium.

GHANNUDI SA and TUFAIL M (1978) A report on a two-day visit to eight salt-water lakes of Ramla Azzallaf Fezzan Libyan Arab Jamahiriya. Libyan J. Sci. 8 69-74.

GREEN AJ, SANCHEZ MI, AMAT F, FIGUEROLA J, HONTARIA F, RUIZ O and HORTAS F (2005) Dispersal of invasive and native brine shrimps Artemia (Anostraca) via waterbirds. Limnol. Oceon. 50 (2) 737-742.

HAMMER UT (1986) Saline Lake Ecosystems of the World. Dr W Junk Publishers Dordrecht, Netherlands. 616 pp.

HONTORIA F and AMAT F (1992) Morphological characterization of adult Artemia (Crustacea Branchiopoda) from different geographical origin Mediterranean populations. J. Plankton Res. 14 949-959.

KING K (2002) On the Use of Artemia as a Vector for Probiotics. Honours Thesis, Rhodes University, Grahamstown, South Africa.

MACDONALD G and BROWN RA (1990) Population dynamics of an asexual brine shrimp Artemia population. J. Exp. Mar. Biol. Ecol. 133 (3) 169-188.

McCARRAHER DB (1972) A preliminary bibliography and lake index of the inland mineral waters of the world. FAO Fish. Circular 146 33.

MITCHELL SA and SEAMAN MT (1988) Observations on the coexistence of fresh and saltwater invertebrates in an inland saltworks. J. Limnol. Soc. S. Afr. 14 121-123.

MONOD T (1969) A propos du Lac des Vers ou Bahr ed-Dud. Inst. Fondam. Afr. Noire. 31 25-41.

PERSOONE G and SORGELOOS P (1980) General aspects of the ecology and biogeography of Artemia. In: Persoone G, Sorgeloos P, Roels O and Jaspers E (eds.) The Brine Shrimp Artemia Vol 3. Ecology Culturing Use in Aquaculture. University Press, Wetteren Belgium. 3-24.

POR FD (1968) Solar Lake on the shores of the Red Sea. Nature 218 $860-861$.

POR FD (1969) Limnology of the heliothermal Solar Lake on the coast of Sinai (Gulf of Eilat). Verhandlungen für Internationale Theoretische und Angewandte Limnologie 17 1031-1034.

RASOWO J and RADULL J (1986) Inoculation of the brine shrimp Artemia salina in Kenya: expected impact on aquaculture development. In: Huisman EA (ed.) Aquaculture Research in the Africa Region. Proc. Afr. Seminar on Aquaculture. International Foundation for Science (IFS) Stockholm Sweden. Kisumu Kenya 7-11 October 1985. Pudoc Wageningen Netherlands. 54-59.

SEAMAN MT, ASHTON PJ and WILLIAMS WD (1991) Inland salt waters of southern Africa. Hydrobiol. 210 75-91. 
SHUMWAY CA (1999) Forgotten Waters: Freshwater and Marine Ecosystems in Africa: Strategies for Biodiversity Conservation and Sustainable Development. Biodiversity Support Program 1999. Boston USA.

SOUSA MI (1994) Artemia in Mozambique. Larviculture and Artemia Newsletter 3327.

TOUMI N, AYADI H, ABID O, CARRIAS J-F, SIME-NGANDO T, BOUKHRIS M, BOUAIN A (2005) Zooplankton distribution in four ponds of different salinity: A seasonal study in the solar systems of Sfax (Tunisia). Hydrobiol. 534 1-9.

TRIANTAPHYLLIDIS GV, ABATZOPOULOS ThJ, MIASA E and SORGELOOS P (1996) International study on Artemia. LVI. Characterization of two Artemia populations from Namibia and Madagascar: cytogenetics biometry hatching characteristics and fatty acid profiles. Hydrobiologia 335 97-106.

TRIANTAPHYLLIDIS GV, CRIEL GRJ, ABATZOPOULOS ThJ and SORGELOOS P (1997a) International study on Artemia. LIV. Morphological study of Artemia with emphasis on Old World strains. II. Parthenogenetic populations. Hydrobiol. 357 155-163.

TRIANTAPHYLLIDIS GV, CRIEL GRJ, ABATZOPOULOS ThJ, THOMAS KM, ELEMAN J, BEARDMORE J and SORGELOOS P (1997b) International study of Artemia. LVII. Morphological and molecular characters suggest con-specificity of all bisexual European and North African Artemia populations. Mar. Biol. $129477-$ 487.

TRIANTAPHYLLIDIS GV, ABATZOPOULOS ThJ and SORGELOOS P (1998) Review of the biogeography of the genus Artemia (Crustacea Anostraca) J. Biogeogr. 25 213-226.

VAN BALLAER ED, VERSICHELE P, LEGER PH, BEN ABDELKADER N, TURKI S and SORGELOOS P (1987) Characterisation of Artemia from different localities in Tunisia with regard to their use in local aquaculture. In: Sorgeloos P, Bengston DA, Decleir W and Jaspers E (eds.) Artemia Research and its Applications. Vol. I. Universal Press, Wetteren Belgium.

VANHAECKE P, TACKAERT W and SORGELOOS P (1987) The biogeography of Artemia: an updated review. In: Sorgeloos P, Bengston DA, Decleir W and Jaspers E (eds.) Artemia Research and its Applications. Vol. 1. Morphology, Genetics, Strain Characterization, Toxicology. Universal Press, Wetteren Belgium. 129-155.

VAN STAPPEN G (2002) Zoogeography. In: Abatzopolous ThJ, Beardmore JA, Clegg JS and Sorgeloos P (eds.) Artemia Basic and Applied Biology. Kluwer Acadamic Publishers, Dordrecht/London/Boston. 171-215.

VU DO QUYNH and NGUYEN NGOC LAM (1987) Inoculation of Artemia in experimental ponds in central Vietnam: an ecological approach and a comparison of three geographical strains. In: Sorgeloos P, Bengston DA, Decleir W and Jaspers E (eds.) Artemia Research and its Applications. Morphology, Genetics, Strain, Characterization, Toxicology. Universal Press, Vol. 1. Wetteren Belgium. 253-269.

WIILIAMS BF and MITCHELL SA (1992) The effect of salinity on the reproductive characteristics of parthenogenetic Artemia from South Africa. Water SA 18 181-184.

WILLIAMS WD (1996) The largest, highest and lowest lakes of the world: Saline lakes. Verhandlungen der Internationalen Vereinigung für Limnologie 26 61-79.

ZEMMOURI A (1991) A note on the genus Artemia in Algeria. Hydrobiol. 212 231-233. 